CRYSTALLOGRAPHIC COMMUNICATIONS

ISSN 2056-9890

Received 31 March 2016

Accepted 6 April 2016

Edited by P. C. Healy, Griffith University, Australia

Keywords: crystal structure; 3-chlorothiophene2-carboxylic acid; acridine.

CCDC reference: 1472507

Supporting information: this article has supporting information at journals.iucr.org/e

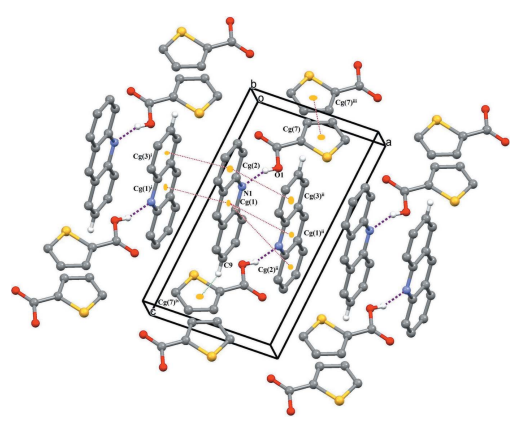

OPEN $\odot$ ACCESS

\section{Supramolecular interactions in a 1:1 co-crystal of acridine and 3-chlorothiophene-2-carboxylic acid}

\author{
Olakkandiyil Prajina, ${ }^{a}$ Packianathan Thomas Muthiah ${ }^{\mathrm{a} *}$ and Franc Perdih ${ }^{\mathrm{b}}$ \\ ${ }^{\mathbf{a}}$ School of Chemistry, Bharathidasan University, Tiruchirappalli 620 024, Tamilnadu, India, and ${ }^{\mathbf{b}}$ Faculty of Chemistry \\ and Chemical Technology, University of Ljubljana, Vecna pot 113, PO Box 537, SI-1000 Ljubljana, Slovenia. \\ *Correspondence e-mail: tommtrichy@yahoo.co.in
}

In the title co-crystal, $\mathrm{C}_{5} \mathrm{H}_{3} \mathrm{ClO}_{2} \mathrm{~S} \cdot \mathrm{C}_{13} \mathrm{H}_{9} \mathrm{~N}$, the components interact with each other via an $\mathrm{O}-\mathrm{H} \cdots \mathrm{N}$ hydrogen bond. Acridine-acridine stacking, thiophenethiophene stacking and acridine-thiophene $\mathrm{C}-\mathrm{H} \cdots \pi$ interactions also occur in the crystal.

\section{Chemical context}

Co-crystals are solids in which two or more molecules crystallize together and interact through non-covalent interactions (Odiase et al., 2015). The study of non-covalent interactions in co-crystals not only adds to our knowledge but also has an undeniable relevance in the context of their pharmaceutical and biological interest (Chakraborty et al., 2014; Desiraju, 1989). The main interactions concerned are various hydrogen bonding, $\pi-\pi$ and $\mathrm{C}-\mathrm{H} \cdots \pi$ interactions (Aakeröy et al., 2010). The acridine molecule is a component present in antihelminthic agents which are used in animals (Durchheimer $e t$ al., 1980). Acridine derivatives also show in vitro activity against protozoa (Ngadi et al., 1993). The acridine group is a well known intercalator interacting with nucleobase pairs (Raju et al., 2016; Nafisi et al., 2007; Sazhnikov et al., 2013). Acridine dyes are also widely used (Solovyeva et al., 2014, Yasarawan et al., 2011). Halogenated thiophene carboxylic acid derivatives are the building blocks of many commercially available insecticides (Hull et al., 2007). We extended our study on supramolecular architectures in acridine molecules with the investigation of the title co-crystal with 3-chlorothiophene-2-carboxylic acid (3TPC).

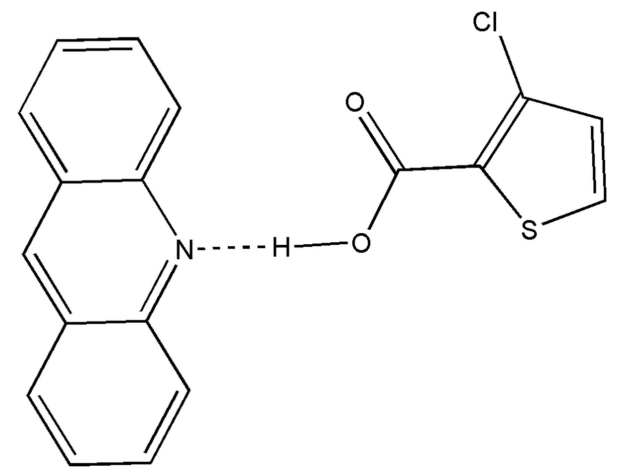

\section{Structural commentary}

The compound (1) is a 1:1 co-crystal of 3TPC and acridine. The internal angle at $\mathrm{N} 1\left[\mathrm{C} 6-\mathrm{N} 1-\mathrm{C} 18=119.30(15)^{\circ}\right]$ and 


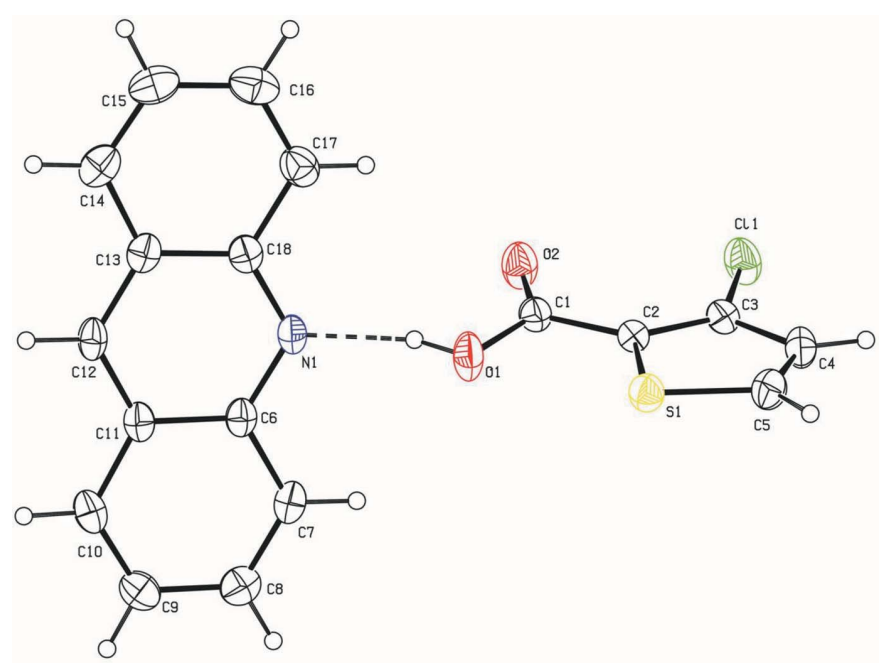

Figure 1

The asymmetric unit of the title compound, showing the atom-numbering scheme. Displacement ellipsoids are drawn at the $50 \%$ probability level. The dashed line represents the $\mathrm{O}-\mathrm{H} \cdots \mathrm{N}$ hydrogen bond.

bond lengths $[\mathrm{C} 18-\mathrm{N} 1=1.346$ (2) and C6-N1 = 1.354 (2) $\mathrm{A}]$ agree with those reported for neutral acridine structures (Aghabozorg et al., 2011; Binder et al., 1982; Goeta et al., 2002). The two external bond angles at the carbon atom of the carboxyl group are $124.13(17)$ and $110.75(15)^{\circ}$. The high
Table 1

Hydrogen-bond geometry $\left(\AA,^{\circ}\right)$.

$\mathrm{Cg} 7$ is the centroid of the thiophene ring.

\begin{tabular}{lllll}
\hline$D-\mathrm{H} \cdots A$ & $D-\mathrm{H}$ & $\mathrm{H} \cdots A$ & $D \cdots A$ & $D-\mathrm{H} \cdots A$ \\
\hline $\mathrm{O} 1-\mathrm{H} 1 \cdots \mathrm{N} 1$ & 0.82 & 1.83 & $2.615(2)$ & 159 \\
$\mathrm{C} 9-\mathrm{H} 9 \cdots C g 7^{\mathrm{i}}$ & 0.93 & 2.94 & $3.773(2)$ & 150 \\
\hline
\end{tabular}

Symmetry code: (i) $-x+1,-y+1,-z+1$.

discrepancy between these two angles is typical of an unionized carboxyl group. The $\mathrm{C}=\mathrm{O}$ distance of 1.316 (2) $\AA$ and C-OH distance of 1.199 (2) $\AA$ are also typical of the carboxyl group. These values also agree with the carboxylic acids reported in the literature (Kowalska et al., 2015; SienkiewiczGromiuk et al., 2016). The dihedral angle between the carboxylic acid group and the thiophene ring is $9.01(13)^{\circ}$. The bond distances and angles involving the thiophene ring agree with those in structures reported earlier (Zhang et al., 2014).

\section{Supramolecular features}

The 3TPC and acridine moieties are linked by an $\mathrm{O}-\mathrm{H} \cdots \mathrm{N}$ hydrogen-bonding interaction between $(\mathrm{O} 1-\mathrm{H} 1)$ of the carboxyl group and the acridine nitrogen atom (N1) (Table 1 and Fig. 1). This $\mathrm{O}-\mathrm{H} \cdots \mathrm{N}$ hydrogen bond is reminiscent of

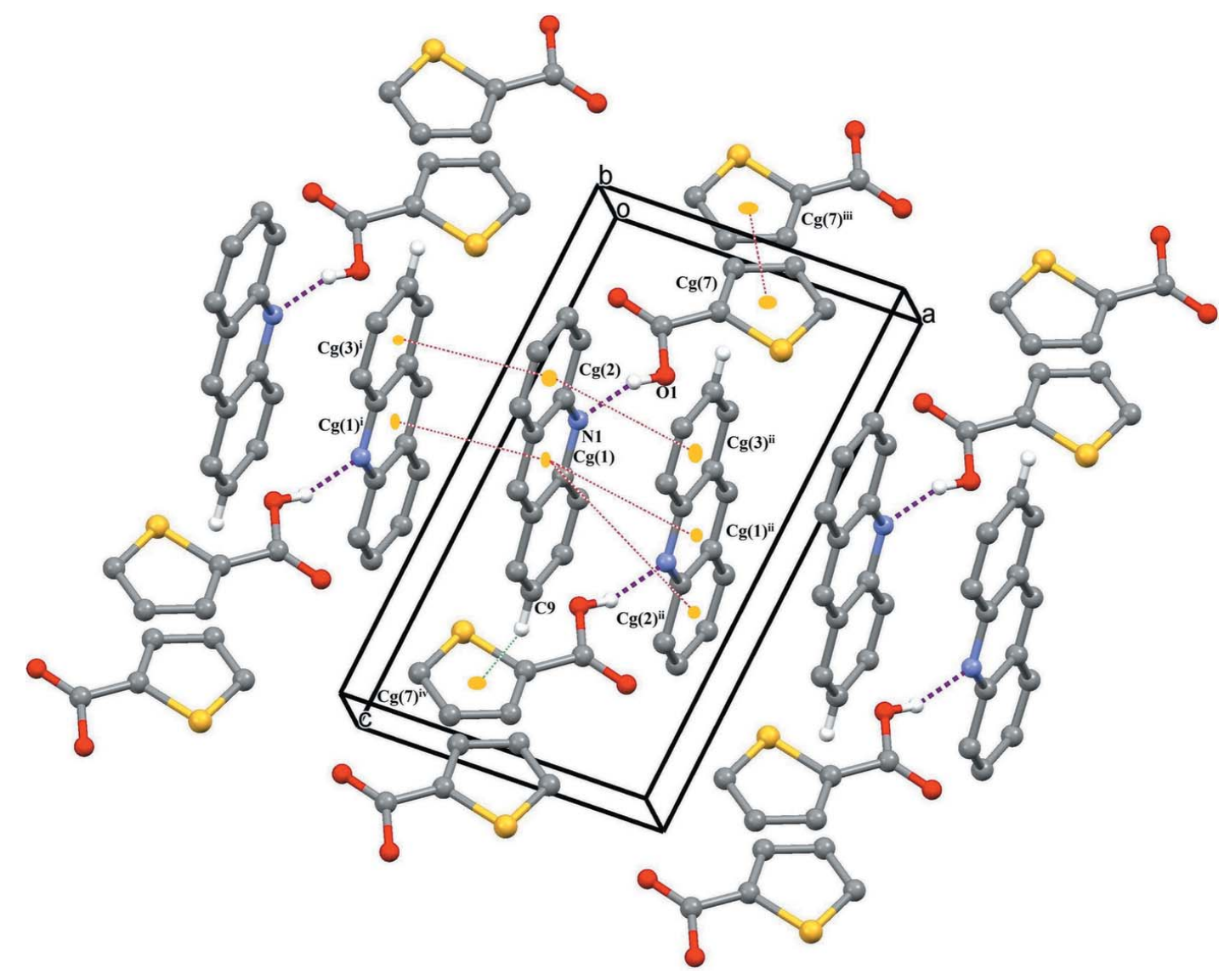

Figure 2

A view of the $\mathrm{O}-\mathrm{H} \cdots \mathrm{N}$ hydrogen bonds (purple dashed lines), $\pi-\pi$ stacking (acridine-acridine and thiophene-thiophene; red dashed lines) and $\mathrm{C}-$ $\mathrm{H} \cdots \pi$ interactions between the acridine $\mathrm{C}-\mathrm{H}$ group and the $\pi$-system of thiophene (green dashed lines). 
the frequently used supramolecular synthon in crystal engineering involving a carboxylic acid and a pyridine molecule (Seaton, 2014; Lemmerer \& Bernstein, 2010; Thomas et al., 2010). A similar type of supramolecular synthon is observed in a series of nine co-crystals involving acridine and benzoic acids (Kowalska et al., 2015). This supramolecular synthon is also present in the co-crystal of 5-chlorothiophene2-carboxylic acid and acridine reported from our laboratory (Jennifer \& Muthiah, 2014). This co-crystal and the title cocrystal differ only in the position of chlorine in the thiophene ring. The hydrogen-bonded units are linked via $\pi-\pi$ stacking interactions between the aromatic systems of acridine molecules $\left[C g 1 \cdots C g 1^{\mathrm{i}}=3.6419(9), C g 1 \cdots C g 1^{\mathrm{ii}}=3.7526(9)\right.$, $C g 1 \cdots C g 2^{\mathrm{ii}}=3.7293(12), C g 2 \cdots C g 3^{\mathrm{i}}=3.6748(12)$ and $C g 2 \cdots C g 3^{\mathrm{ii}}=3.7298(12) \AA$ where $C g 1$ is the centroid of the $\mathrm{N} 1 / \mathrm{C} 6 / \mathrm{C} 11 / \mathrm{C} 12 / \mathrm{C} 13 / \mathrm{C} 18 \mathrm{ring}, C g 2$ is the centroid of the C6$\mathrm{C} 11$ ring and $\mathrm{Cg} 3$ is the centroid of the $\mathrm{C} 13-\mathrm{C} 18$ ring; symmetry codes: (i) $-x, 2-y, 1-z$; (ii) $1-x, 2-y, 1-z]$ and between the thiophene rings $\left[C g 7 \cdots C g 7^{\mathrm{iii}}=3.7611\right.$ (12) $\AA$ where $C g 7$ is the centroid of the thiophene ring; symmetry code: (iii) $1-x, 1-y,-z]$. The crystal structure also features $\mathrm{C}-\mathrm{H} \cdots \pi$ interactions, forming a three-dimensional supramolecular architecture (Table 1 and Fig. 2).

\section{Database survey}

The crystal structures of a number of acridine co-crystals, acridinium salts and their metal complexes have been investigated in a variety of crystalline environments such as diphenic acid-acridine (1:1) (Shaameri et al., 2001a), 4,4'bis(hydroxyazobenzene)-acridine (Chakraborty et al., 2014), orcinol-acridine (1:2) and orcinol-acridine (1:1) co-crystal hydrate (Mukherjee et al., 2011), acridinium isophthalate (Shaameri et al., 2001b) and acridinium 6-carboxypyridine-2carboxylate monohydrate (Derikvand et al., 2011). A variety of metal complexes of acridine have also been reported (Ha, 2010, 2012; Sloufova \& Slouf, 2000, 2001).

\section{Synthesis and crystallization}

To $10 \mathrm{ml}$ of a hot methanol solution of 3TPC (40.6 mg, $25 \mathrm{mmol}$ ) were added $10 \mathrm{ml}$ of a hot methanolic solution of acridine (44.8 mg, $25 \mathrm{mmol}$ ). The resulting solution was warmed over a water bath for half an hour and then kept at room temperature for crystallization. After a week yellow plate-like crystals of (1) were obtained.

\section{Refinement}

Crystal data, data collection and structure refinement details are summarized in Table 2. All hydrogen atoms were readily located in difference Fourier maps and were subsequently treated as riding atoms in geometrically idealized positions, with $\mathrm{C}-\mathrm{H}=0.93$ and $\mathrm{O}-\mathrm{H}=0.82 \AA$, and with $U_{\text {iso }}(\mathrm{H})=$ $k U_{\text {eq }}(\mathrm{C}, \mathrm{O})$, where $k=1.5$ for hydroxy and 1.2 for all other $\mathrm{H}$ atoms.
Table 2

Experimental details.

\begin{tabular}{|c|c|}
\hline \multicolumn{2}{|l|}{ Crystal data } \\
\hline Chemical formula & $\mathrm{C}_{5} \mathrm{H}_{3} \mathrm{ClO}_{2} \mathrm{~S} \cdot \mathrm{C}_{13} \mathrm{H}_{9} \mathrm{~N}$ \\
\hline$M_{\mathrm{r}}$ & 341.80 \\
\hline Crystal system, space group & Triclinic, $P \overline{1}$ \\
\hline Temperature $(\mathrm{K})$ & 293 \\
\hline$a, b, c(\AA)$ & $7.3371(4), 8.3286(5), 13.3819(8)$ \\
\hline$\alpha, \beta, \gamma\left({ }^{\circ}\right)$ & $107.577(5), 97.706(5), 93.953(5)$ \\
\hline$V\left(\AA^{3}\right)$ & $767.32(8)$ \\
\hline$Z$ & 2 \\
\hline Radiation type & Мо $K \alpha$ \\
\hline$\mu\left(\mathrm{mm}^{-1}\right)$ & 0.39 \\
\hline Crystal size $(\mathrm{mm})$ & $0.60 \times 0.30 \times 0.10$ \\
\hline \multicolumn{2}{|l|}{ Data collection } \\
\hline Diffractometer & $\begin{array}{l}\text { Agilent SuperNova Dual Source } \\
\text { diffractometer with an Atlas } \\
\text { detector }\end{array}$ \\
\hline Absorption correction & $\begin{array}{l}\text { Multi-scan (CrysAlis PRO; } \\
\text { Agilent, 2013) }\end{array}$ \\
\hline$T_{\min }, T_{\max }$ & $0.813,1.000$ \\
\hline $\begin{array}{l}\text { No. of measured, independent and } \\
\text { observed }[I>2 \sigma(I)] \text { reflections }\end{array}$ & $7182,3516,2722$ \\
\hline$R_{\text {int }}$ & 0.022 \\
\hline$(\sin \theta / \lambda)_{\max }\left(\AA^{-1}\right)$ & 0.649 \\
\hline \multicolumn{2}{|l|}{ Refinement } \\
\hline$R\left[F^{2}>2 \sigma\left(F^{2}\right)\right], w R\left(F^{2}\right), S$ & $0.038,0.109,1.02$ \\
\hline No. of reflections & 3516 \\
\hline No. of parameters & 209 \\
\hline $\mathrm{H}$-atom treatment & H-atom parameters constrained \\
\hline$\Delta \rho_{\max }, \Delta \rho_{\min }\left(\mathrm{e} \AA^{-3}\right)$ & $0.21,-0.23$ \\
\hline
\end{tabular}

Computer programs: CrysAlis PRO (Agilent, 2013), SHELXS97 (Sheldrick, 2008), SHELXL2014 (Sheldrick, 2015), PLATON (Spek, 2009) and Mercury (Macrae et al., 2008), PLATON (Spek, 2009) and publCIF (Westrip, 2010).

\section{Acknowledgements}

OKP thanks the UGC-SAP and UGC-BSR India for the award of an RFSMS. PTM is thankful to the UGC, New Delhi, for a UGC-BSR one-time grant to Faculty. FP thanks the Slovenian Research Agency for financial support (P1-02300175), as well as the EN-FIST Centre of Excellence, Slovenia, for use of the SuperNova diffractometer

\section{References}

Aakeröy, C. B., Champness, S. R. \& Janiak, C. (2010). CrystEngComm, 12, 22-43.

Aghabozorg, H., Goodarzi, S., Mirzaei, M. \& Notash, B. (2011). Acta Cryst. E67, o126.

Agilent (2013). CrysAlis PRO. Agilent Technologies UK Ltd, Yarnton, England.

Binder, W., Karl, N. \& Stezowski, J. J. (1982). Acta Cryst. B38, 2915 2916.

Chakraborty, S., Rajput, L. \& Desiraju, G. R. (2014). Cryst. Growth Des. 14, 2571-2577.

Derikvand, Z., Olmstead, M. M. \& Attar Gharamaleki, J. (2011). Acta Cryst. E67, o416.

Desiraju, G. R. (1989). In Crystal engineering: the design of organic solids. Amsterdam: Elsevier.

Durchheimer, W., Raether, W., Seliger, H. \& Seidenath, H. (1980). Arzneim.-Forsch. Drug. Res. 30, 1041-1046.

Goeta, A. E., Lawrence, S. E., Meehan, M. M., O'Dowd, A. \& Spalding, T. R. (2002). Polyhedron, 21, 1689-1694.

Ha, K. (2010). Acta Cryst. E66, m1083.

Ha, K. (2012). Acta Cryst. E68, o196. 
Hull, J. W., Romer, D. R., Podhorez, D. E., Ash, M. L. \& Brady, C. H. (2007). Beilstein J. Org. Chem. 3, 23.

Jennifer, S. J. \& Muthiah, P. T. (2014). Chem. Cent. J. 8, 20.

Kowalska, K., Trzybiński, D. \& Sikorski, A. (2015). CrystEngComm, 17, 7199-7212.

Lemmerer, A. \& Bernstein, J. (2010). CrystEngComm, 12, 2029-2033.

Macrae, C. F., Bruno, I. J., Chisholm, J. A., Edgington, P. R., McCabe, P., Pidcock, E., Rodriguez-Monge, L., Taylor, R., van de Streek, J. \& Wood, P. A. (2008). J. Appl. Cryst. 41, 466-470.

Mukherjee, A., Grobelny, P., Thakur, T. S. \& Desiraju, G. R. (2011). Cryst. Growth Des. 11, 2637-2653.

Nafisi, S., Saboury, A. A., Keramat, N., Neault, J. F. \& Tajmir-Riahi, H. A. (2007). J. Mol. Struct. 827, 35-43.

Ngadi, L., Bsiri, N., Mahamoud, A., Galy, A. M., Galy, J. P., Soyfer, J. C., Barbe, J., Placidi, M., Rodriguez-Santiago, J. J., Mesa-Valle, C., Lombardo, R., Mascaro, C. \& Osuna, A. (1993). Arzneim.-Forsch. Drug. Res. 43, 480-483.

Odiase, I., Nicholson, C. E., Ahmad, R., Cooper, J., Yufit, D. S. \& Cooper, S. J. (2015). Acta Cryst. C71, 276-283.

Raju, G., Vishwanath, S., Prasad, A., Patel, B. K. \& Prabusankar, G. (2016). J. Mol. Struct. 1107, 291-299.

Sazhnikov, V. A., Khlebunov, A. A., Sazonov, S. K., Vedernikov, A. I., Safonov, A. A., Bagatur'yants, A. A., Kuz'mina, L. G., Howard, J. A. K., Gromov, S. P. \& Alfimov, M. V. (2013). J. Mol. Struct. 1053, 79-88.
Seaton, C. C. (2014). CrystEngComm, 16, 5878-5886.

Shaameri, Z., Shan, N. \& Jones, W. (2001a). Acta Cryst. E57, o107501077.

Shaameri, Z., Shan, N. \& Jones, W. (2001b). Acta Cryst. E57, o945o946.

Sheldrick, G. M. (2008). Acta Cryst. A64, 112-122.

Sheldrick, G. M. (2015). Acta Cryst. C71, 3-8.

Sienkiewicz-Gromiuk, J., Tarasiuk, B. \& Mazur, L. (2016). J. Mol. Struct. 1110, 65-71.

Sloufova, I. \& Slouf, M. (2000). Acta Cryst. C56, 1312-1313.

Sloufova, I. \& Slouf, M. (2001). Acta Cryst. C57, 248-249.

Solovyeva, E. V., Myund, L. A., Starova, G. L., Dem'yanchuk, E. M., Makarov, A. A. \& Denisova, A. S. (2014). J. Mol. Struct. 1063, 235241.

Spek, A. L. (2009). Acta Cryst. D65, 148-155.

Thomas, L. H., Blagden, N., Gutmann, M. J., Kallay, A. A., Parkin, A., Seaton, C. C. \& Wilson, C. C. (2010). Cryst. Growth Des. 10, 2770 2774.

Westrip, S. P. (2010). J. Appl. Cryst. 43, 920-925.

Yasarawan, N., Thipyapong, K. \& Ruangpornvisuti, V. (2011). J. Mol. Struct. 1006, 635-641.

Zhang, Q., Luo, J., Ye, L., Wang, H., Huang, B., Zhang, J., Wu, J., Zhang, S. \& Tian, Y. (2014). J. Mol. Struct. 1074, 33-42. 


\section{supporting information}

Acta Cryst. (2016). E72, 659-662 [doi:10.1107/S2056989016005685]

Supramolecular interactions in a 1:1 co-crystal of acridine and 3-chlorothiophene-2-carboxylic acid

\section{Olakkandiyil Prajina, Packianathan Thomas Muthiah and Franc Perdih}

Computing details

Data collection: CrysAlis PRO (Agilent, 2013); cell refinement: CrysAlis PRO (Agilent, 2013); data reduction: CrysAlis PRO (Agilent, 2013); program(s) used to solve structure: SHELXS97 (Sheldrick, 2008); program(s) used to refine structure: SHELXL2014 (Sheldrick, 2015); molecular graphics: PLATON (Spek, 2009) and Mercury (Macrae et al., 2008); software used to prepare material for publication: PLATON (Spek, 2009) and publCIF (Westrip, 2010).

Acridine-3-chlorothiophene-2-carboxylic acid (1/1)

Crystal data

$\mathrm{C}_{5} \mathrm{H}_{3} \mathrm{ClO}_{2} \mathrm{~S} \cdot \mathrm{C}_{13} \mathrm{H}_{9} \mathrm{~N}$

$M_{r}=341.80$

Triclinic, $P \overline{1}$

$a=7.3371$ (4) $\AA$

$b=8.3286(5) \AA$

$c=13.3819(8) \AA$

$\alpha=107.577(5)^{\circ}$

$\beta=97.706(5)^{\circ}$

$\gamma=93.953(5)^{\circ}$

$V=767.32(8) \AA^{3}$

\section{Data collection}

Agilent SuperNova Dual Source diffractometer with an Atlas detector

Radiation source: SuperNova (Mo) X-ray Source

Detector resolution: 10.4933 pixels $\mathrm{mm}^{-1}$

$\omega$ scans

Absorption correction: multi-scan

(CrysAlis PRO; Agilent, 2013)

$T_{\min }=0.813, T_{\max }=1.000$

\section{Refinement}

Refinement on $F^{2}$

Least-squares matrix: full

$R\left[F^{2}>2 \sigma\left(F^{2}\right)\right]=0.038$

$w R\left(F^{2}\right)=0.109$

$S=1.02$

3516 reflections

209 parameters

0 restraints
$Z=2$
$F(000)=352$
$D_{\mathrm{x}}=1.479 \mathrm{Mg} \mathrm{m}^{-3}$

Mo $K \alpha$ radiation, $\lambda=0.71073 \AA$

Cell parameters from 2635 reflections

$\theta=3.9-29.2^{\circ}$

$\mu=0.39 \mathrm{~mm}^{-1}$

$T=293 \mathrm{~K}$

Plate, yellow

$0.60 \times 0.30 \times 0.10 \mathrm{~mm}$

7182 measured reflections

3516 independent reflections

2722 reflections with $I>2 \sigma(I)$

$R_{\text {int }}=0.022$

$\theta_{\text {max }}=27.5^{\circ}, \theta_{\min }=2.8^{\circ}$

$h=-9 \rightarrow 8$

$k=-10 \rightarrow 10$

$l=-17 \rightarrow 17$

Hydrogen site location: inferred from neighbouring sites

$\mathrm{H}$-atom parameters constrained

$w=1 /\left[\sigma^{2}\left(F_{\mathrm{o}}{ }^{2}\right)+(0.0488 P)^{2}+0.133 P\right]$

where $P=\left(F_{\mathrm{o}}^{2}+2 F_{\mathrm{c}}{ }^{2}\right) / 3$

$(\Delta / \sigma)_{\max }<0.001$

$\Delta \rho_{\max }=0.21 \mathrm{e} \AA^{-3}$

$\Delta \rho_{\min }=-0.23 \mathrm{e}^{-3}$ 


\section{Special details}

Geometry. All esds (except the esd in the dihedral angle between two 1.s. planes) are estimated using the full covariance matrix. The cell esds are taken into account individually in the estimation of esds in distances, angles and torsion angles; correlations between esds in cell parameters are only used when they are defined by crystal symmetry. An approximate (isotropic) treatment of cell esds is used for estimating esds involving l.s. planes.

Fractional atomic coordinates and isotropic or equivalent isotropic displacement parameters $\left(\hat{A}^{2}\right)$

\begin{tabular}{|c|c|c|c|c|}
\hline & $x$ & $y$ & $z$ & $U_{\text {iso }} * / U_{\text {eq }}$ \\
\hline Cl1 & $0.21228(7)$ & $0.16757(7)$ & -0.02749 (4) & $0.05748(17)$ \\
\hline $\mathrm{S} 1$ & $0.69521(6)$ & $0.48402(6)$ & $0.14957(4)$ & $0.04516(15)$ \\
\hline O1 & 0.42177 (19) & $0.63242(19)$ & $0.26579(11)$ & $0.0581(4)$ \\
\hline H1 & 0.3486 & 0.6911 & 0.2968 & $0.087^{*}$ \\
\hline $\mathrm{O} 2$ & $0.16569(18)$ & $0.49072(19)$ & $0.15614(11)$ & $0.0569(4)$ \\
\hline N1 & $0.26273(19)$ & $0.85354(18)$ & $0.39993(11)$ & $0.0390(3)$ \\
\hline $\mathrm{C} 1$ & $0.3309(2)$ & $0.5158(2)$ & $0.18026(14)$ & $0.0392(4)$ \\
\hline $\mathrm{C} 2$ & $0.4606(2)$ & $0.4179(2)$ & $0.11735(13)$ & $0.0366(4)$ \\
\hline $\mathrm{C} 3$ & $0.4277(2)$ & $0.2754(2)$ & $0.03048(14)$ & $0.0403(4)$ \\
\hline $\mathrm{C} 4$ & $0.5886(3)$ & $0.2201(3)$ & $-0.00919(16)$ & $0.0499(5)$ \\
\hline H4 & 0.5890 & 0.1248 & -0.0675 & $0.060^{*}$ \\
\hline $\mathrm{C} 5$ & $0.7426(3)$ & $0.3226(3)$ & $0.04802(16)$ & $0.0512(5)$ \\
\hline H5 & 0.8614 & 0.3061 & 0.0331 & $0.061^{*}$ \\
\hline $\mathrm{C} 6$ & $0.2995(2)$ & $0.8560(2)$ & $0.50232(14)$ & $0.0367(4)$ \\
\hline $\mathrm{C} 7$ & $0.3599(2)$ & $0.7110(2)$ & $0.52526(16)$ & $0.0451(4)$ \\
\hline H7 & 0.3756 & 0.6162 & 0.4701 & $0.054^{*}$ \\
\hline $\mathrm{C} 8$ & $0.3948(3)$ & $0.7096(3)$ & $0.62689(17)$ & $0.0508(5)$ \\
\hline H8 & 0.4331 & 0.6131 & 0.6407 & $0.061 *$ \\
\hline C9 & $0.3742(3)$ & $0.8518(3)$ & $0.71176(16)$ & $0.0505(5)$ \\
\hline H9 & 0.3994 & 0.8485 & 0.7811 & $0.061^{*}$ \\
\hline $\mathrm{C} 10$ & $0.3179(3)$ & $0.9933(3)$ & $0.69378(15)$ & $0.0470(5)$ \\
\hline H10 & 0.3050 & 1.0864 & 0.7508 & $0.056^{*}$ \\
\hline $\mathrm{C} 11$ & $0.2784(2)$ & $1.0008(2)$ & $0.58805(14)$ & $0.0374(4)$ \\
\hline $\mathrm{C} 12$ & $0.2206(2)$ & $1.1415(2)$ & $0.56483(14)$ & $0.0406(4)$ \\
\hline H12 & 0.2070 & 1.2375 & 0.6197 & $0.049^{*}$ \\
\hline $\mathrm{C} 13$ & $0.1826(2)$ & $1.1413(2)$ & $0.45997(15)$ & $0.0397(4)$ \\
\hline $\mathrm{C} 14$ & $0.1250(3)$ & $1.2834(3)$ & $0.43165(18)$ & $0.0521(5)$ \\
\hline H14 & 0.1088 & 1.3814 & 0.4843 & $0.063^{*}$ \\
\hline $\mathrm{C} 15$ & $0.0936(3)$ & $1.2765(3)$ & $0.3283(2)$ & $0.0606(6)$ \\
\hline H15 & 0.0577 & 1.3706 & 0.3104 & $0.073^{*}$ \\
\hline $\mathrm{C} 16$ & $0.1148(3)$ & $1.1285(3)$ & 0.24777 (19) & $0.0631(6)$ \\
\hline H16 & 0.0921 & 1.1263 & 0.1773 & $0.076^{*}$ \\
\hline $\mathrm{C} 17$ & $0.1677(3)$ & $0.9888(3)$ & $0.27050(16)$ & $0.0528(5)$ \\
\hline H17 & 0.1788 & 0.8915 & 0.2159 & $0.063^{*}$ \\
\hline $\mathrm{C} 18$ & $0.2061(2)$ & $0.9916(2)$ & $0.37835(14)$ & $0.0398(4)$ \\
\hline
\end{tabular}


Atomic displacement parameters $\left(\AA^{2}\right)$

\begin{tabular}{lllllll}
\hline & $U^{11}$ & $U^{22}$ & $U^{33}$ & $U^{12}$ & $U^{13}$ & $U^{23}$ \\
\hline C11 & $0.0514(3)$ & $0.0565(3)$ & $0.0477(3)$ & $-0.0036(2)$ & $0.0025(2)$ & $-0.0040(2)$ \\
S1 & $0.0407(3)$ & $0.0458(3)$ & $0.0437(3)$ & $0.00472(19)$ & $0.00429(19)$ & $0.0075(2)$ \\
O1 & $0.0474(8)$ & $0.0552(9)$ & $0.0509(8)$ & $0.0084(7)$ & $0.0069(6)$ & $-0.0141(7)$ \\
O2 & $0.0397(8)$ & $0.0638(10)$ & $0.0539(9)$ & $0.0063(6)$ & $0.0081(6)$ & $-0.0012(7)$ \\
N1 & $0.0361(8)$ & $0.0383(8)$ & $0.0363(8)$ & $0.0043(6)$ & $0.0094(6)$ & $0.0008(7)$ \\
C1 & $0.0435(10)$ & $0.0365(10)$ & $0.0355(9)$ & $0.0045(7)$ & $0.0057(7)$ & $0.0085(8)$ \\
C2 & $0.0401(9)$ & $0.0374(10)$ & $0.0318(9)$ & $0.0077(7)$ & $0.0050(7)$ & $0.0096(8)$ \\
C3 & $0.0442(10)$ & $0.0398(10)$ & $0.0339(9)$ & $0.0052(7)$ & $0.0035(7)$ & $0.0082(8)$ \\
C4 & $0.0549(12)$ & $0.0478(12)$ & $0.0415(10)$ & $0.0136(9)$ & $0.0119(9)$ & $0.0027(9)$ \\
C5 & $0.0465(11)$ & $0.0576(13)$ & $0.0505(12)$ & $0.0165(9)$ & $0.0154(9)$ & $0.0132(10)$ \\
C6 & $0.0282(8)$ & $0.0371(10)$ & $0.0405(10)$ & $0.0006(6)$ & $0.0098(7)$ & $0.0049(8)$ \\
C7 & $0.0423(10)$ & $0.0379(10)$ & $0.0516(11)$ & $0.0065(8)$ & $0.0148(8)$ & $0.0057(9)$ \\
C8 & $0.0464(11)$ & $0.0514(12)$ & $0.0594(13)$ & $0.0073(9)$ & $0.0118(9)$ & $0.0227(11)$ \\
C9 & $0.0502(11)$ & $0.0574(13)$ & $0.0436(11)$ & $-0.0018(9)$ & $0.0069(8)$ & $0.0175(10)$ \\
C10 & $0.0491(11)$ & $0.0457(11)$ & $0.0376(10)$ & $-0.0037(8)$ & $0.0092(8)$ & $0.0015(9)$ \\
C11 & $0.0303(8)$ & $0.0360(9)$ & $0.0386(9)$ & $-0.0029(7)$ & $0.0078(7)$ & $0.0016(8)$ \\
C12 & $0.0339(9)$ & $0.0341(10)$ & $0.0432(10)$ & $-0.0011(7)$ & $0.0092(7)$ & $-0.0038(8)$ \\
C13 & $0.0273(8)$ & $0.0377(10)$ & $0.0497(11)$ & $-0.0001(7)$ & $0.0074(7)$ & $0.0074(8)$ \\
C14 & $0.0380(10)$ & $0.0440(12)$ & $0.0718(14)$ & $0.0030(8)$ & $0.0063(9)$ & $0.0158(11)$ \\
C15 & $0.0440(11)$ & $0.0639(15)$ & $0.0809(17)$ & $0.0051(10)$ & $0.0024(11)$ & $0.0366(14)$ \\
C16 & $0.0498(12)$ & $0.0863(18)$ & $0.0580(14)$ & $0.0021(11)$ & $0.0013(10)$ & $0.0343(14)$ \\
C17 & $0.0444(11)$ & $0.0654(14)$ & $0.0438(11)$ & $0.0043(9)$ & $0.0050(8)$ & $0.0116(10)$ \\
C18 & $0.0278(8)$ & $0.0465(11)$ & $0.0405(10)$ & $0.0012(7)$ & $0.0059(7)$ & $0.0075(8)$ \\
& & & & & & \\
& & & & & &
\end{tabular}

Geometric parameters $\left(\AA,{ }^{\circ}\right)$

\begin{tabular}{llll}
\hline $\mathrm{C} 11-\mathrm{C} 3$ & $1.7207(18)$ & $\mathrm{C} 8-\mathrm{H} 8$ & 0.9300 \\
$\mathrm{~S} 1-\mathrm{C} 5$ & $1.692(2)$ & $\mathrm{C} 9-\mathrm{C} 10$ & $1.352(3)$ \\
$\mathrm{S} 1-\mathrm{C} 2$ & $1.7261(17)$ & $\mathrm{C} 9-\mathrm{H} 9$ & 0.9300 \\
$\mathrm{O} 1-\mathrm{C} 1$ & $1.316(2)$ & $\mathrm{C} 10-\mathrm{C} 11$ & $1.427(3)$ \\
$\mathrm{O} 1-\mathrm{H} 1$ & 0.8200 & $\mathrm{C} 10-\mathrm{H} 10$ & 0.9300 \\
$\mathrm{O} 2-\mathrm{C} 1$ & $1.199(2)$ & $\mathrm{C} 11-\mathrm{C} 12$ & $1.379(2)$ \\
$\mathrm{N} 1-\mathrm{C} 18$ & $1.346(2)$ & $\mathrm{C} 12-\mathrm{C} 13$ & $1.393(2)$ \\
$\mathrm{N} 1-\mathrm{C} 6$ & $1.354(2)$ & $\mathrm{C} 12-\mathrm{H} 12$ & 0.9300 \\
$\mathrm{C} 1-\mathrm{C} 2$ & $1.478(3)$ & $\mathrm{C} 13-\mathrm{C} 14$ & $1.421(3)$ \\
$\mathrm{C} 2-\mathrm{C} 3$ & $1.368(3)$ & $\mathrm{C} 13-\mathrm{C} 18$ & $1.426(3)$ \\
$\mathrm{C} 3-\mathrm{C} 4$ & $1.408(3)$ & $\mathrm{C} 14-\mathrm{C} 15$ & $1.355(3)$ \\
$\mathrm{C} 4-\mathrm{C} 5$ & $1.353(3)$ & $\mathrm{C} 14-\mathrm{H} 14$ & 0.9300 \\
$\mathrm{C} 4-\mathrm{H} 4$ & 0.9300 & $\mathrm{C} 15-\mathrm{C} 16$ & 0.9300 \\
$\mathrm{C} 5-\mathrm{H} 5$ & $\mathrm{C} 15-\mathrm{H} 15$ & $1.357(3)$ \\
$\mathrm{C} 6-\mathrm{C} 7$ & 0.9300 & $\mathrm{C} 16-\mathrm{C} 17$ & 0.9300 \\
$\mathrm{C} 6-\mathrm{C} 11$ & $1.418(2)$ & $\mathrm{C} 16-\mathrm{H} 16$ & $1.426(3)$ \\
$\mathrm{C} 7-\mathrm{C} 8$ & $1.426(2)$ & $\mathrm{C} 17-\mathrm{C} 18$ & 0.9300 \\
$\mathrm{C} 7-\mathrm{H} 7$ & $1.354(3)$ & $\mathrm{C} 17-\mathrm{H} 17$ & \\
$\mathrm{C} 8-\mathrm{C} 9$ & 0.9300 & &
\end{tabular}




\begin{tabular}{|c|c|c|c|}
\hline $\mathrm{C} 5-\mathrm{S} 1-\mathrm{C} 2$ & $92.22(9)$ & $\mathrm{C} 8-\mathrm{C} 9-\mathrm{H} 9$ & 119.6 \\
\hline $\mathrm{C} 1-\mathrm{O} 1-\mathrm{H} 1$ & 109.5 & $\mathrm{C} 9-\mathrm{C} 10-\mathrm{C} 11$ & $120.52(19)$ \\
\hline $\mathrm{C} 18-\mathrm{N} 1-\mathrm{C} 6$ & $119.31(15)$ & $\mathrm{C} 9-\mathrm{C} 10-\mathrm{H} 10$ & 119.7 \\
\hline $\mathrm{O} 2-\mathrm{C} 1-\mathrm{O} 1$ & $125.12(18)$ & $\mathrm{C} 11-\mathrm{C} 10-\mathrm{H} 10$ & 119.7 \\
\hline $\mathrm{O} 2-\mathrm{C} 1-\mathrm{C} 2$ & $124.13(17)$ & $\mathrm{C} 12-\mathrm{C} 11-\mathrm{C} 6$ & $118.43(16)$ \\
\hline $\mathrm{O} 1-\mathrm{C} 1-\mathrm{C} 2$ & $110.75(15)$ & $\mathrm{C} 12-\mathrm{C} 11-\mathrm{C} 10$ & $123.13(17)$ \\
\hline $\mathrm{C} 3-\mathrm{C} 2-\mathrm{C} 1$ & $130.54(16)$ & $\mathrm{C} 6-\mathrm{C} 11-\mathrm{C} 10$ & $118.44(17)$ \\
\hline $\mathrm{C} 3-\mathrm{C} 2-\mathrm{S} 1$ & $109.54(14)$ & $\mathrm{C} 11-\mathrm{C} 12-\mathrm{C} 13$ & $120.66(17)$ \\
\hline $\mathrm{C} 1-\mathrm{C} 2-\mathrm{S} 1$ & $119.91(13)$ & $\mathrm{C} 11-\mathrm{C} 12-\mathrm{H} 12$ & 119.7 \\
\hline $\mathrm{C} 2-\mathrm{C} 3-\mathrm{C} 4$ & $113.99(17)$ & $\mathrm{C} 13-\mathrm{C} 12-\mathrm{H} 12$ & 119.7 \\
\hline $\mathrm{C} 2-\mathrm{C} 3-\mathrm{Cl} 1$ & $124.70(15)$ & $\mathrm{C} 12-\mathrm{C} 13-\mathrm{C} 14$ & $122.99(18)$ \\
\hline $\mathrm{C} 4-\mathrm{C} 3-\mathrm{C} 11$ & $121.31(15)$ & $\mathrm{C} 12-\mathrm{C} 13-\mathrm{C} 18$ & $117.77(16)$ \\
\hline $\mathrm{C} 5-\mathrm{C} 4-\mathrm{C} 3$ & $111.64(19)$ & $\mathrm{C} 14-\mathrm{C} 13-\mathrm{C} 18$ & $119.23(17)$ \\
\hline $\mathrm{C} 5-\mathrm{C} 4-\mathrm{H} 4$ & 124.2 & $\mathrm{C} 15-\mathrm{C} 14-\mathrm{C} 13$ & $120.2(2)$ \\
\hline $\mathrm{C} 3-\mathrm{C} 4-\mathrm{H} 4$ & 124.2 & $\mathrm{C} 15-\mathrm{C} 14-\mathrm{H} 14$ & 119.9 \\
\hline $\mathrm{C} 4-\mathrm{C} 5-\mathrm{S} 1$ & $112.61(15)$ & $\mathrm{C} 13-\mathrm{C} 14-\mathrm{H} 14$ & 119.9 \\
\hline $\mathrm{C} 4-\mathrm{C} 5-\mathrm{H} 5$ & 123.7 & $\mathrm{C} 14-\mathrm{C} 15-\mathrm{C} 16$ & $120.6(2)$ \\
\hline $\mathrm{S} 1-\mathrm{C} 5-\mathrm{H} 5$ & 123.7 & $\mathrm{C} 14-\mathrm{C} 15-\mathrm{H} 15$ & 119.7 \\
\hline $\mathrm{N} 1-\mathrm{C} 6-\mathrm{C} 7$ & $119.41(16)$ & $\mathrm{C} 16-\mathrm{C} 15-\mathrm{H} 15$ & 119.7 \\
\hline $\mathrm{N} 1-\mathrm{C} 6-\mathrm{C} 11$ & $121.65(16)$ & $\mathrm{C} 17-\mathrm{C} 16-\mathrm{C} 15$ & $121.5(2)$ \\
\hline $\mathrm{C} 7-\mathrm{C} 6-\mathrm{C} 11$ & $118.94(16)$ & $\mathrm{C} 17-\mathrm{C} 16-\mathrm{H} 16$ & 119.2 \\
\hline $\mathrm{C} 8-\mathrm{C} 7-\mathrm{C} 6$ & $120.47(19)$ & $\mathrm{C} 15-\mathrm{C} 16-\mathrm{H} 16$ & 119.2 \\
\hline $\mathrm{C} 8-\mathrm{C} 7-\mathrm{H} 7$ & 119.8 & $\mathrm{C} 16-\mathrm{C} 17-\mathrm{C} 18$ & $119.8(2)$ \\
\hline $\mathrm{C} 6-\mathrm{C} 7-\mathrm{H} 7$ & 119.8 & $\mathrm{C} 16-\mathrm{C} 17-\mathrm{H} 17$ & 120.1 \\
\hline $\mathrm{C} 7-\mathrm{C} 8-\mathrm{C} 9$ & $120.91(19)$ & $\mathrm{C} 18-\mathrm{C} 17-\mathrm{H} 17$ & 120.1 \\
\hline $\mathrm{C} 7-\mathrm{C} 8-\mathrm{H} 8$ & 119.5 & $\mathrm{~N} 1-\mathrm{C} 18-\mathrm{C} 17$ & $119.21(18)$ \\
\hline $\mathrm{C} 9-\mathrm{C} 8-\mathrm{H} 8$ & 119.5 & $\mathrm{~N} 1-\mathrm{C} 18-\mathrm{C} 13$ & $122.18(16)$ \\
\hline $\mathrm{C} 10-\mathrm{C} 9-\mathrm{C} 8$ & $120.73(18)$ & $\mathrm{C} 17-\mathrm{C} 18-\mathrm{C} 13$ & $118.61(17)$ \\
\hline $\mathrm{C} 10-\mathrm{C} 9-\mathrm{H} 9$ & 119.6 & & \\
\hline $\mathrm{O} 2-\mathrm{C} 1-\mathrm{C} 2-\mathrm{C} 3$ & $-7.7(3)$ & $\mathrm{C} 7-\mathrm{C} 6-\mathrm{C} 11-\mathrm{C} 12$ & $179.62(14)$ \\
\hline $\mathrm{O} 1-\mathrm{C} 1-\mathrm{C} 2-\mathrm{C} 3$ & $171.99(17)$ & $\mathrm{N} 1-\mathrm{C} 6-\mathrm{C} 11-\mathrm{C} 10$ & $179.36(15)$ \\
\hline $\mathrm{O} 2-\mathrm{C} 1-\mathrm{C} 2-\mathrm{S} 1$ & $170.91(14)$ & $\mathrm{C} 7-\mathrm{C} 6-\mathrm{C} 11-\mathrm{C} 10$ & $-0.4(2)$ \\
\hline $\mathrm{O} 1-\mathrm{C} 1-\mathrm{C} 2-\mathrm{S} 1$ & $-9.4(2)$ & $\mathrm{C} 9-\mathrm{C} 10-\mathrm{C} 11-\mathrm{C} 12$ & $179.99(16)$ \\
\hline $\mathrm{C} 5-\mathrm{S} 1-\mathrm{C} 2-\mathrm{C} 3$ & $0.03(13)$ & $\mathrm{C} 9-\mathrm{C} 10-\mathrm{C} 11-\mathrm{C} 6$ & $0.0(3)$ \\
\hline $\mathrm{C} 5-\mathrm{S} 1-\mathrm{C} 2-\mathrm{C} 1$ & $-178.87(14)$ & $\mathrm{C} 6-\mathrm{C} 11-\mathrm{C} 12-\mathrm{C} 13$ & $0.5(2)$ \\
\hline $\mathrm{C} 1-\mathrm{C} 2-\mathrm{C} 3-\mathrm{C} 4$ & $178.94(16)$ & $\mathrm{C} 10-\mathrm{C} 11-\mathrm{C} 12-\mathrm{C} 13$ & $-179.48(15)$ \\
\hline $\mathrm{S} 1-\mathrm{C} 2-\mathrm{C} 3-\mathrm{C} 4$ & $0.2(2)$ & $\mathrm{C} 11-\mathrm{C} 12-\mathrm{C} 13-\mathrm{C} 14$ & $-179.16(15)$ \\
\hline $\mathrm{C} 1-\mathrm{C} 2-\mathrm{C} 3-\mathrm{C} 11$ & $-1.6(3)$ & $\mathrm{C} 11-\mathrm{C} 12-\mathrm{C} 13-\mathrm{C} 18$ & $-0.2(2)$ \\
\hline $\mathrm{S} 1-\mathrm{C} 2-\mathrm{C} 3-\mathrm{C} 11$ & $179.68(10)$ & $\mathrm{C} 12-\mathrm{C} 13-\mathrm{C} 14-\mathrm{C} 15$ & $178.67(17)$ \\
\hline $\mathrm{C} 2-\mathrm{C} 3-\mathrm{C} 4-\mathrm{C} 5$ & $-0.4(2)$ & $\mathrm{C} 18-\mathrm{C} 13-\mathrm{C} 14-\mathrm{C} 15$ & $-0.2(3)$ \\
\hline $\mathrm{C} 11-\mathrm{C} 3-\mathrm{C} 4-\mathrm{C} 5$ & $-179.89(13)$ & $\mathrm{C} 13-\mathrm{C} 14-\mathrm{C} 15-\mathrm{C} 16$ & $0.9(3)$ \\
\hline $\mathrm{C} 3-\mathrm{C} 4-\mathrm{C} 5-\mathrm{S} 1$ & $0.4(2)$ & $\mathrm{C} 14-\mathrm{C} 15-\mathrm{C} 16-\mathrm{C} 17$ & $-0.3(3)$ \\
\hline $\mathrm{C} 2-\mathrm{S} 1-\mathrm{C} 5-\mathrm{C} 4$ & $-0.25(16)$ & $\mathrm{C} 15-\mathrm{C} 16-\mathrm{C} 17-\mathrm{C} 18$ & $-1.1(3)$ \\
\hline $\mathrm{C} 18-\mathrm{N} 1-\mathrm{C} 6-\mathrm{C} 7$ & $-179.75(14)$ & $\mathrm{C} 6-\mathrm{N} 1-\mathrm{C} 18-\mathrm{C} 17$ & $179.93(15)$ \\
\hline $\mathrm{C} 18-\mathrm{N} 1-\mathrm{C} 6-\mathrm{C} 11$ & $0.5(2)$ & $\mathrm{C} 6-\mathrm{N} 1-\mathrm{C} 18-\mathrm{C} 13$ & $-0.2(2)$ \\
\hline $\mathrm{N} 1-\mathrm{C} 6-\mathrm{C} 7-\mathrm{C} 8$ & $-179.02(16)$ & $\mathrm{C} 16-\mathrm{C} 17-\mathrm{C} 18-\mathrm{N} 1$ & $-178.39(17)$ \\
\hline
\end{tabular}




$\begin{array}{llll}\mathrm{C} 11-\mathrm{C} 6-\mathrm{C} 7-\mathrm{C} 8 & 0.7(2) & \mathrm{C} 16-\mathrm{C} 17-\mathrm{C} 18-\mathrm{C} 13 & 1.8(3) \\ \mathrm{C} 6-\mathrm{C} 7-\mathrm{C} 8-\mathrm{C} 9 & -0.7(3) & \mathrm{C} 12-\mathrm{C} 13-\mathrm{C} 18-\mathrm{N} 1 & 0.1(2) \\ \mathrm{C} 7-\mathrm{C} 8-\mathrm{C} 9-\mathrm{C} 10 & 0.3(3) & \mathrm{C} 14-\mathrm{C} 13-\mathrm{C} 18-\mathrm{N} 1 & 179.06(14) \\ \mathrm{C} 8-\mathrm{C} 9-\mathrm{C} 10-\mathrm{C} 11 & 0.0(3) & \mathrm{C} 12-\mathrm{C} 13-\mathrm{C} 18-\mathrm{C} 17 & 179.95(15) \\ \mathrm{N} 1-\mathrm{C} 6-\mathrm{C} 11-\mathrm{C} 12 & -0.6(2) & \mathrm{C} 14-\mathrm{C} 13-\mathrm{C} 18-\mathrm{C} 17 & -1.1(2)\end{array}$

Hydrogen-bond geometry $\left(A,{ }^{\circ}\right)$

$\mathrm{Cg} 7$ is the centroid of the thiophene ring.

\begin{tabular}{lllll}
\hline$D-\mathrm{H} \cdots A$ & $D-\mathrm{H}$ & $\mathrm{H} \cdots A$ & $D \cdots A$ & $D-\mathrm{H} \cdots A$ \\
\hline $\mathrm{O} 1-\mathrm{H} 1 \cdots \mathrm{N} 1$ & 0.82 & 1.83 & $2.615(2)$ & 159 \\
$\mathrm{C} 9-\mathrm{H} 9 \cdots C g 7^{\mathrm{i}}$ & 0.93 & 2.94 & $3.773(2)$ & 150
\end{tabular}

Symmetry code: (i) $-x+1,-y+1,-z+1$. 